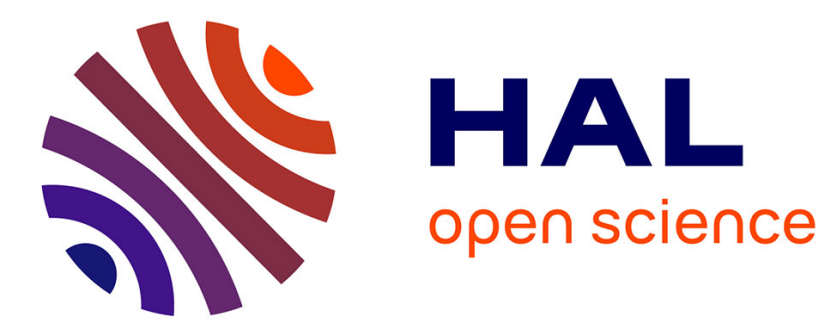

\title{
Surface, forme et croissance des mineraux des argiles
}

Julia Henin, J. Chaussidon, Raoul Calvet

\section{To cite this version:}

Julia Henin, J. Chaussidon, Raoul Calvet. Surface, forme et croissance des mineraux des argiles. BULLETIN DU GROUPE FRANCAIS DES ARGILES, 1969, 21, pp.31-45. hal-02730243

\section{HAL Id: hal-02730243 \\ https://hal.inrae.fr/hal-02730243}

Submitted on 2 Jun 2020

HAL is a multi-disciplinary open access archive for the deposit and dissemination of scientific research documents, whether they are published or not. The documents may come from teaching and research institutions in France or abroad, or from public or private research centers.
L'archive ouverte pluridisciplinaire HAL, est destinée au dépôt et à la diffusion de documents scientifiques de niveau recherche, publiés ou non, émanant des établissements d'enseignement et de recherche français ou étrangers, des laboratoires publics ou privés. 
Bull. Groupe franç. Argiles, t. XXI, p. 31-45

SURFACE, FORME ET GROISSANCE DES

MINERAUX DES ARGILES

Par S. HENIN, J. CHAUSSIDON, R. CALVET

Laboratoire des Sols - C.N.R.A. - 78 - Versailles

Résumé - La discussion de la notion de surface spécifique des minéraux phylliteux conduit à un examen approfondi des propriétés géométriques des particules élémentaires. Leur forme, leurs dimensions et la surface de ces minéraux sont étroitement reliées. Il apparaft cependant que la valeur de la surface spécifique est' déterminée par le mode d'assemblage des particules. Il est alors possible d'essayer d'entrevoir les relations entre cette propriété et les modalités de la cristallisation.

Abstract - The discussion about the notion of specific surface in clay minerals leads to a thoroughly examination of the geometric properties of elemental particles. Their shape, size and surface are strongly connected. However, it appears that the value of specific surface is determined by the mode of assemblage of particles. Then, it is possibl t to attempt forseeing the relations between this property and the modalities of crystallization.

\section{I. - INTRODUCTION}

Le concept de surface spécifique possède pratiquement deux sens. C'est d'une part la surface totale des particules contenues dans l'unité de poids ou de volume d'un échantillon donnè. Mais d'autre part, en ce qui concerne les minéraux argileux, on estime que l'ordre de grandeur de la surface spécifique constitue une sorte de caractéristique d'une espèce ou d'une 
farilile de minéraux; dăns ce sens, une deuxième notion de spécificité est attachée au concept.

Nous nous proposons de revenir sur les calculs et les hypothèses qui sont à la base de l'évaluation des surfaces et qui permettent de les comparer à d'autres caractéristiques de ces matériaux. Bien qu'il s'agisse d'opérations très élémentaires, elles présentent néanmoins divers aspects et c'est pourquoi il nous a paru utile d'en faire une présentation d'ensemble. A partir de ces premières conclusions nous pourrons aborder le troisième thème de notre communication, c'est-à-dire la croissance, car ce sont les particularités de ce phénomène qui peuvent faire de la valeur de la surface spécifique une donnée caractéristique du minéral.

\section{II. - FORME ET SURFACE DES PARTICULES}

Partons de la formule la plus générale permettant d'établir la surface totale des particules constituant un échantillon quelconque d'argile, celles-ci étant supposées de-même dimension et de même forme.

$$
\begin{array}{ll}
\mathrm{S}(\text { totale) } & =\mathrm{n} \text { } \\
\mathrm{S} & =\text { surface d'une particule } \\
\mathrm{n} & =\text { nombre de particules }
\end{array}
$$

Comme il n'est généralement pas techniquement possible de mesurer s et souvent difficile de déterminer $n$, on transforme cettc formulc in tonant compte du fait que : 


$$
n=\frac{P}{p}=\frac{d V}{d v}
$$

où, PV sont respectivement le poids total et le volume réel total de l'échantillon, $d$ la densité, $p$ et $v$ le poids et le volume d'une particule.

Introduite dans la formule (1) cette relation permet d'écrire :

$$
\frac{S}{P}=\frac{s}{p} \quad \text { (3) et } \quad \frac{S}{V}=\frac{s}{v}
$$

Si l'on ramène $P$ ou $V$ à 1 , la surface $S$ correspondante est alors une surface spécifique que nous écrirons $S_{P}$ et $S_{V}$.

Cette même considération nous amène à écrire :

$$
n_{p}=\frac{1}{p} \quad \text { (4) } \quad \text { et } \quad n_{v}=\frac{1}{v}
$$

L'existence de cette dernière relation montre que si nous parlons de surface spécifique, il revient au même de multiplier la surface élémentaire par le nombre spécifique de particules, ou de la diviser par le volume unitaire d'une particule.

L'application de ces formules classiques nécessite une hypothìso sur la forme des particules, si l'on veut écrire une relation entre s at $v$. 
ce cui ramène lá mesur a de la surface spécifique à celle d’une dimension. C'est ¿insi que la surface totale de 1'échantillon pour des particuies sphériques est $S_{u}=\frac{6}{D}$ et pour des cubes. Su $=\frac{6}{a}$, D désignant le diamètre de la sphère et a liarête du cube exprimśs eñ centimètres.

Le choix de modèles isodimensionnels se justifie pour deux raisons. C'est, d'une part la condition pour réduire le problème à la mesure d'une dimension, et c'est d'autre partque ies méthodes de séparation fon appel soit à la formule de STOKES, soit aux résultats du tamisage, ce qui implique dans les deux cas., ou bien que les yarticules soient conformes au modèle, ou qu'elles en soient suffisamment proches pour que l'on puisse évaluer un rayon considéré comme "moyen" ou "efficace".

Il faut reconnaitre qu'il est très difficile de s'affranchir de ces hypotizèses, qui correspondent non seulement à toute une série d'opérations classiques, mais qui permettent par des calculs simples d'appliquer leurs résultats à la prévision de certaines propriétés telles que la vitesse de filtration du matériau (formule de CARMAN-KOZENY) Ia vitesse de dépôt d'une suspension, etc...

Par contre, le caractère dissymétrique permet d'évaluer d'autres propriétés liées à la constitution cristallochimique des argiles, par exemple de distinguer les phénomènes se manifestant sur les surfaces basales ou sur les surfaces latérales des cristallites. Il permet également de comprendrc la structure et les propriétés des gels.

Pour relier la surface et la forme en tenant compte de la dissymitriu. nous nous référerons à un modèle. cclui du prismẹ droit. 
Soit $x$ y $z$ les longueurs des trois arêtes

$s=2(x y+y z+x z)$

et, en tenant compte de (3') et (4') $s_{u}=\frac{2(x y+y z+x z)}{x y z}$

La condition $n . v=1$ nous conduit à poser $x y z \leqslant 1$.

Physiquement parlant, cette relation signifie que le cube de dimension 1, par exemple $1 \mathrm{~cm}^{3}$, peut être, ou ciivisé en n particules élémentaires, ou constitué d'une seụle particule qui peut être un cube ou dans les cas extrêmes, une plaquette extrêmement mince, ou une fibre très allongée, ce qui implique un comportement particulier, tel que, par exemple, celui d'une matière plastique. Bien que ces propriétés ne soient pas celles de nos matériaux à l'échelle des particules, considérons quelques applications de cette équation, celles-ci nous permettant d'examiner l'ensemble des cas possibles. Cette étude met également en évidence le sens des opérations arithmétiques effectuées sur les données numériques fournies par l'expérience. Nous supposerons que $\mathrm{x}=\mathrm{y}$, c'est-à-dire que deux des faces sont carrées. Déns cette hypothèse :

Surface latérale $\left(s_{1}\right)$ d'une particiıle $=4 \mathrm{xz}$

Surface latérale totale $=\frac{4 x z}{x^{2} z}=\frac{4}{x}$

Surface basale $\left(s_{b}\right)$ d'une particule $=2 x^{2}$

Surfáce basale totale $=\frac{2 x^{2}}{x^{2} z}=\frac{2}{z}$ 
L'équation (5) devient alors :

$$
S u=\frac{2 x^{2}+4 x z}{x^{2} z}
$$

qui peut encore s'écrire :

$$
S u=\left(\frac{2}{z}+\frac{4}{x}\right)
$$

Bien qu'équivalente mathématiquement, cette dernière équation (5') représente le résultat de la division d'un cube par des plans équidistants, parallèles à ses façes, il faut alors introduire la condition $x, z \leqslant 1$, nous nous rapprocherions ainsi de certaines exigences propres à nos matériaux.

Mais on peut également tirer de l'équation (5) les relations suivantes :

$$
x=\frac{4 z}{z S u-2} \quad \text { (6). } \quad \text { et } \quad z=\frac{2 x}{x S u-4}
$$

Ces fonctions sont des hyperboles équilatères ayant comme asymptotes $\mathbf{x}=\frac{4}{\mathrm{Su}}$ et $\mathbf{z}=\frac{2}{\mathrm{Su}}$. Ces cas limites caractérisent des particules très allongées, ou des plaquettes très minces. L'existence de ces solutions asymptotiques et la forme de la courbe montrent que ces résultats sont tròs stables, c'est-à-dire que la valeur de $z$ ou de $x$ ne varie plus que très lentement à partir d'une certaine dissymétrie. Ces propriétés correspondent i1 un nombre de particules variables et dont l'épaisseur peut être réduite sans tonir compto dos limites imposées par la structure. L'exemple le plus typique d'un tol comportement serait celui d'un corps plastique. 
On peut alors calculer $v$ et $n$ en fonction de $\mathrm{Su}$

$$
v=x^{2} z=\frac{2 x^{3}}{x S u-4} \quad \text { et } \quad n=\frac{x S u-4}{2 x^{3}}
$$

L'étude de la variation de cette fonction, dans le domaine où elle a un sens physique $\left(\frac{4}{S u}<x<\curvearrowright\right)$, montre que pour une valeur donnée de $S u, n$ passe par un maximum $N$ pour $x=\frac{6}{S u}$, d'où $N=\frac{S^{3}}{216}$ qui s'applique évidemment à des particules cubiques. La figure 1 correspondant à $S u=f(N)$ délimite le domaine de toutes les valeurs de $n$ compatibles avec une valeur donnée de Su. Cette figure montre en particulier qu'avec un matériau plastique on pourrait avoir toutes les valeurs possibles de $n$ entre 1 et $N$, pour une même surface.

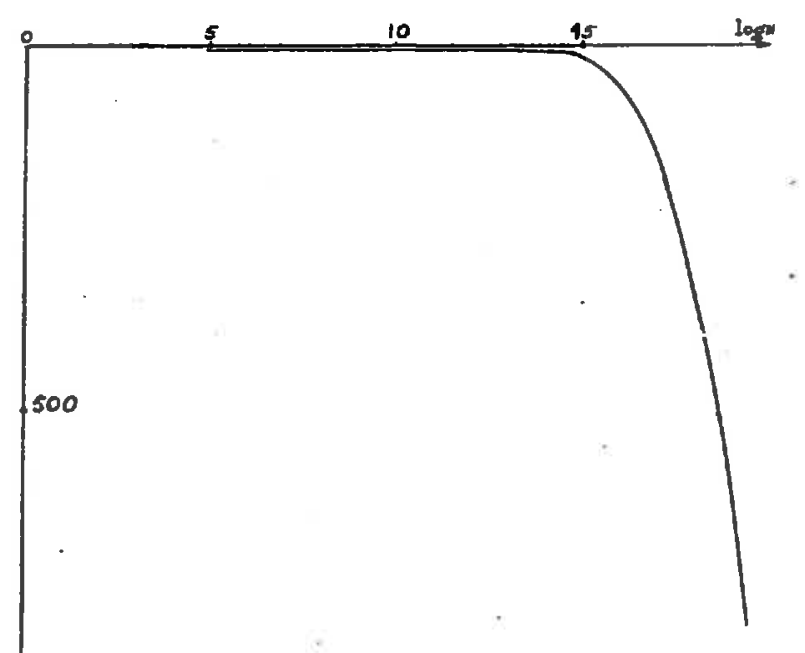

\section{Figure 1}

Courbe $s_{u} m^{2} / g=f(N$ maxi) 
Si nous passons au cas réel, il faut tenir compte du fait que l'épaisseur z correspondant à un feuillet élémentaire est constante. Si l'on introduit cette condition dans la formule ( 7 ), on constate qu'il n'y a qu'une valeur possible de $x$ pour une valeur de Su, si bien que, d'après cette équa tion, l'évolution d'un système réel ayant cette propriété n'est pas exprimable. Par contre, si nous as socions entre eux les cubes élémentaires pour en faire une plaquette, la surface basale $2 x^{2}$ reste constante; c'est la surface latérale qui varie, l'accolement des particules les unes aux autres la faisant progressivenent disparaftre, ce qui provoque nécessairement une réduction de la surface totale.

Comme le comportement de nos matériaux ne correspond pas à celui impliqué par les équations précédentes, nous reprendrons les calculs sur d'autres bases. Prenons comme élément de base la maille et décrivons la variation des surfaces en fonction de ses dimensions, de manière à ce que celles ci constituent des données limites.

En tenant compte de $\left(4^{1}\right), N_{\mathrm{v}}=\frac{1}{\mathrm{v}} \cdot \mathrm{N}_{\mathrm{v}}$ est le nombre maximum de particules possibles, puisque celles-ci correspondent à 1 maille élémentaire de volume $v . N_{v}$ a cependant le sens d'un nombre spécifique (rapport à l'unité de volume). Les surfaces des particules réelles seront exprimées en tenant compte du fait qu'elles sont constituées de $N_{I}$ mailles dans le plan et, le cas échéant, de $N_{2}$ feuillets de $N_{1}$ mailles superposées. Le nombre de particules
est $\mathrm{n}_{\mathrm{v}}=\frac{\mathrm{N}_{\mathrm{v}}}{\mathrm{N}_{\mathrm{l}}}$ pour un assemblage dans le plan, et $\mathrm{n}_{\mathrm{v}}=\frac{\mathrm{N}_{\mathrm{v}}}{\mathrm{N}_{1} \cdot \mathrm{N}_{2}}$ s'il y a assemblage dans le plan et dans l'espace. Il nous faut alors prendre pour les différents types de juxtaposition possible le produit $S u=n_{v} s$ et $S_{p}=\cdot \frac{n_{v} . s}{d}$. 
1) Caaㅡㄹ des plaquettes
(assemblage suivant deux dimensions - montmorillonite)

On part de $\mathrm{N}_{\mathrm{v}}$ mailles prismatiques qui sont associées côte à côte de manière à constituer $\mathrm{n}_{\mathrm{v}}$ particules. Les arêtes ont respectivement $5 \times 9 \times 10 \AA$.

$s=\left(2 N_{1} s_{b}+2 \sqrt{N_{1}} s_{2 N_{1}}+2 \sqrt{N_{1}} s_{2}\right)$ soit, pour les $N_{v}$ mailles du volume unitaire, $S u=\frac{2 N_{\mathrm{V}}}{N_{1}}\left(N_{1} s_{b}+\sqrt{N}_{1}^{2} s_{1}+\sqrt{N}_{1} s_{2}\right)$, en prenant une densité de 2,5 et les valeurs des paramètres indiquées pour la maille on calcule :

$$
\mathrm{Spm} \mathrm{m}^{2} / \mathrm{g}=17,8\left(45+\frac{140}{\sqrt{\mathrm{N}_{1}}}\right)
$$

La figure 2 représente les variations de $\mathrm{Sp}$ en fonction de la plus grande dimension du cristallite; on constate que pratiquement cette valeur est voisine de $800 \mathrm{~m}^{2} / \mathrm{g}$, ce qui signifie que, pour un minéral ayant un feuilles de $10 \AA$ d'épaisseur et dont toutes les surfaces sont accessibles à un réactif comme le glycol, la surface totale de l'échantillon est pratiquement constante. Le résultat est donc valable même si l'échantillon est plus ou moins polydisperse, ce qui permet d'étendre les conclusions à des systèmes réels. 

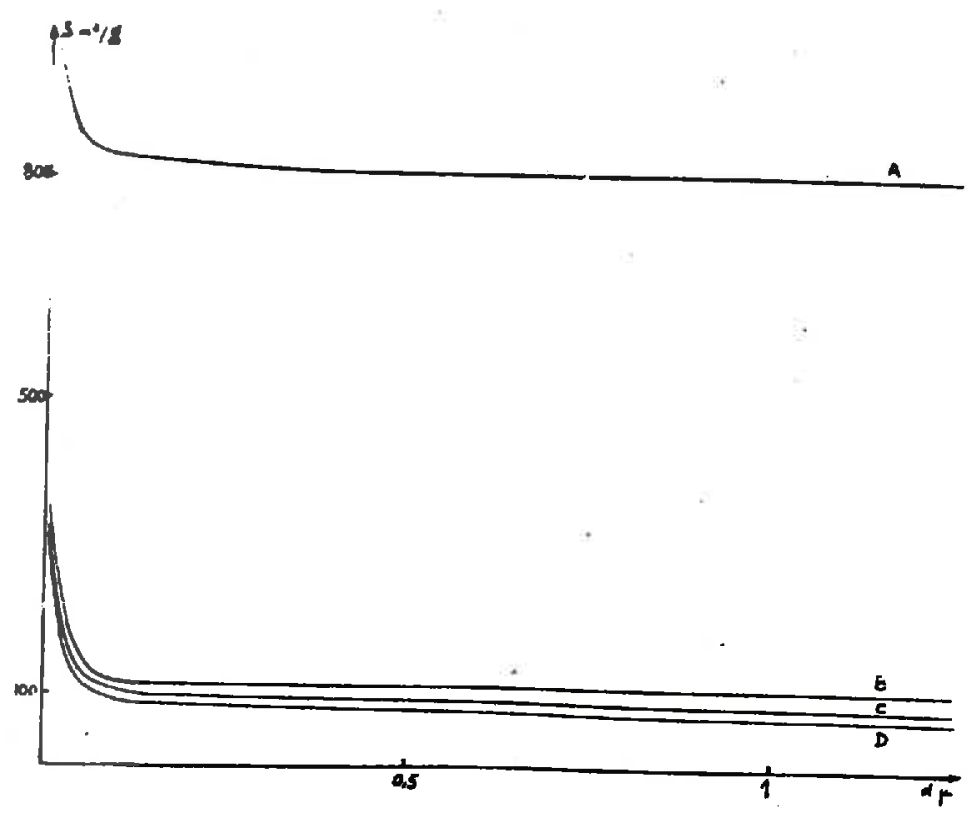

Figure 2

Courbe $\mathrm{Sm}^{2} / \mathrm{g}=\mathrm{f} \mathrm{d} \mu$ ( $\mathrm{d}=$ plus grande dimension de la particule). Cas de la montmorillonite ou de l'illite. Epaisseur des cristallites : $\mathrm{A}=10 \AA ; \quad \mathrm{B}=80 \AA ; \quad \mathrm{C}=100 \AA ; \mathrm{D}=120 \AA$.

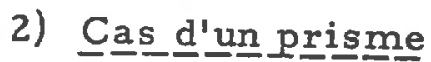

(assemblage suivant trois dimensions - kaolinite)

On prend alors comme côté du prisme droit élémentaire les valeurs 5,9 et $7 \AA$.

$$
S u=\frac{2 N_{v}}{N_{1} N_{2}}\left(N_{1} s_{b}+N_{2} \sqrt{N_{1}}\left(s l_{1}+s_{2}\right)\right.
$$


$\mathrm{N}_{1}$ étant le nombre de mailles associées dans le plan et $\mathrm{N}_{2}$ le nombre de feuillets élémentaires juxtaposés.

Dans ces conditions, $s_{b} n^{\prime}$ est plus constante comme dans le cas précédent. Par ailleurs $s_{p}=25,4\left(\frac{25}{N_{2}}+\frac{98}{\sqrt{N_{1}}}\right)$, avec $d=2,5$.

La figure 3 correspond à cẹ cas. Là encore les valeurs asymptotiques montrent que le minéral est nécessairement épais, d'après les surfaces spécifiques indiquées, cette dimension ne devrait pas être inférieure à $350 \AA$ et le cas est applicable aux systèmes polydisperses. On peut étendre ce calcul aux cas des illites et des cristallites de montmorillonites telles que les mesures de surface B.E.T. les mettent en évidence. Les résultats correspondent aux courbes $B, C$ et $D$ de la figure 2 .

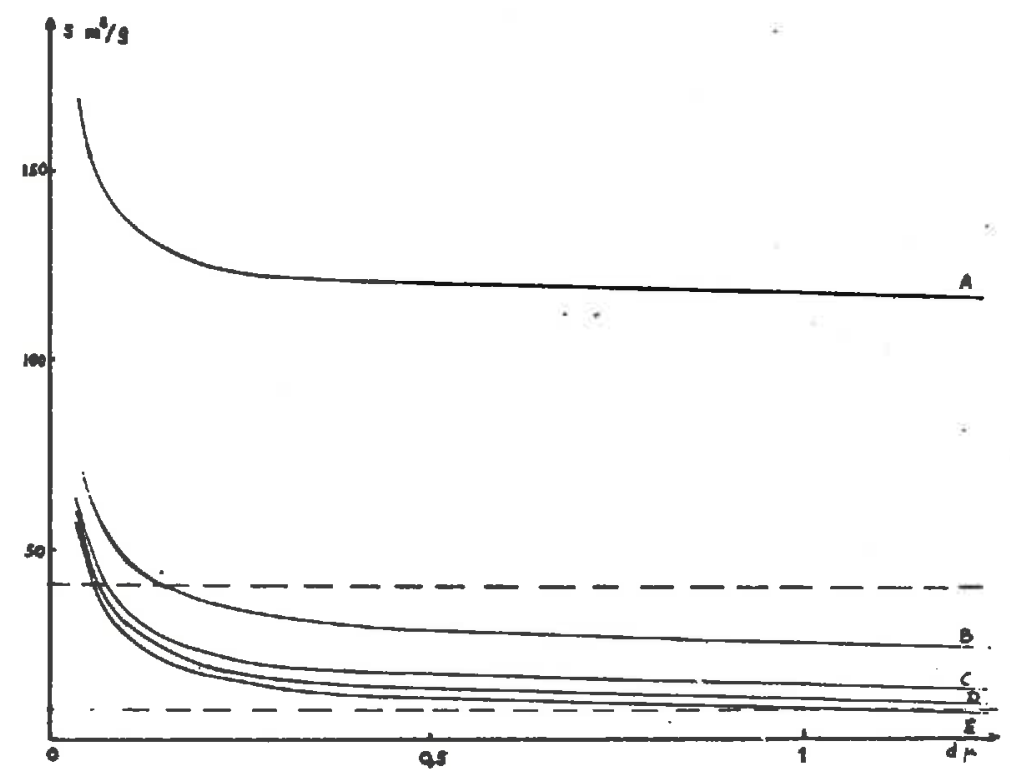

\section{Figure 3}

(An:bes $\mathrm{m}^{2} \mathrm{~g}=\mathrm{fd} \mu$. ( $\mathrm{d}=$ plus grande dimension de la particulo).

$\therefore$ he lit kaolinite. F.paisseur des cristallites: $A=70 \AA$;

$13 \quad$ inti $\& ; C:=00 \AA ; D=750 \AA ; E: 1000 \AA$.

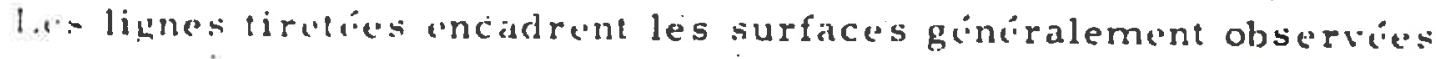
pour la kaolinita. 
3) Cás des fibres

(assemblages suivant une direction - attapulgites, sépiolites)

La maille a cette fois comme dimensions 5,18 et $13 \AA$.

$$
S u=2 N_{v}\left(\frac{s 1_{2}}{N_{1}}+\frac{s 1_{1}+s_{b}}{N_{2}}\right) .
$$

et $\quad S_{p}=6,84\left(\frac{234}{N_{1}}+\frac{155}{N_{2}}\right)$

$N_{1}$ exprime le nombre de mailles associées dans une section et

$\mathrm{N}_{2}$ le nombre de sections associées suivant l'axe de la fibre.

Les courbes de la figure 4 représentent les variations de la surface pour différentes longueurs de fibres en fonction de la plus grande dimension de la section, ici encore les surfaces observées expérimentalement impliquent une longueur.de fibre minimum et dépendent assez peu de la section. Le système peut donc être polydisperse.

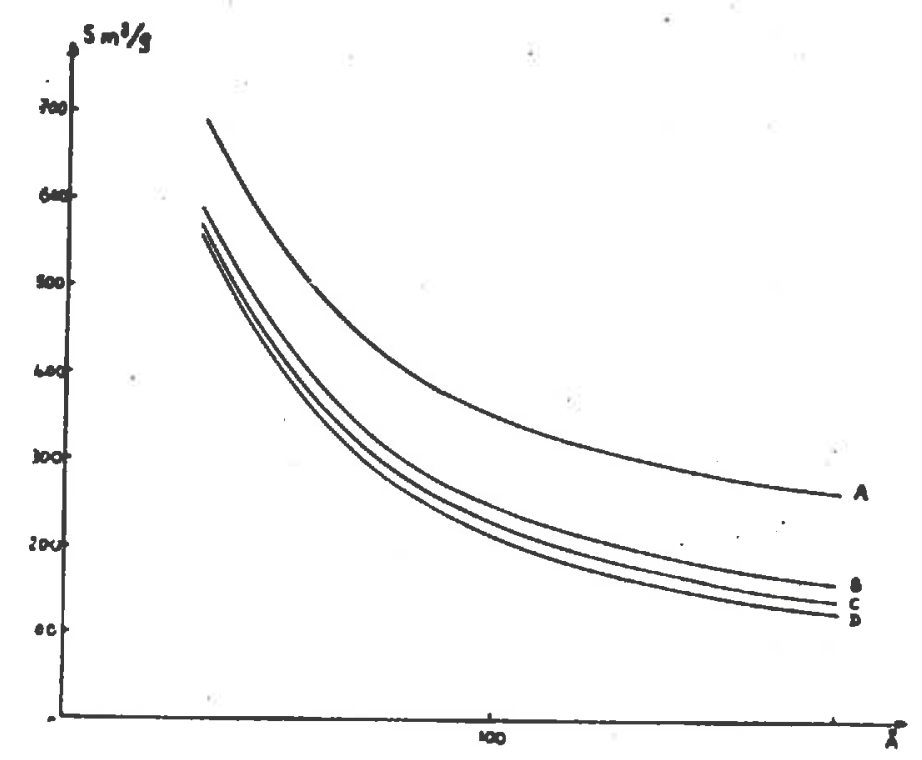

\section{Figure 4}

Courbe $\mathrm{Sm}^{2} / \mathrm{g}=\mathrm{fd} \mu(\mathrm{d}=$ plus grande dimension de la particule). Cas de l'at. tapulgite. Longueur des fibres: $A=50 \AA ; B=150 \AA$; $C=250 \AA ; D=350 \AA$. 


\section{II. - CONSIDERATIONS SUR LA CROISSANCE}

,

On constate donc que 1'existence des relations hyperboliques impliquée par la notion physique de surface spécifique a pour conséquence des résultats très stables, qui sont déterminés par l'existence d'une dimension particulière; c'est donc par ce biais que l'ordre de grandeur de la surface spécifique peut devenir une caractéristique de l'espèce.

Considérons maintenant les conséquences qui en résultent du point de vue de la croissance de ces minéraux. Si la surface spécifique n'était pas caraciéristique de l'espèce, l'échantillon pourrait renfermer des particules ayant toutes les dimensions possibles. Le fait qu'il y ait un ordre de grandeur caractéristique implique donc qu'il y ait peu de germes, c'est-à-dire que la croissance d'une mąsse d'argile s'effectue beaucoup plus par le grossissement des particules existantes que par l'apparition de nouveaux germes. Cette condition est d'autant plus acceptable que dès que les particules ont atteint une certaine taille, du fait de leur dissymétrie, l'augmentation de celle-ci ne modifie

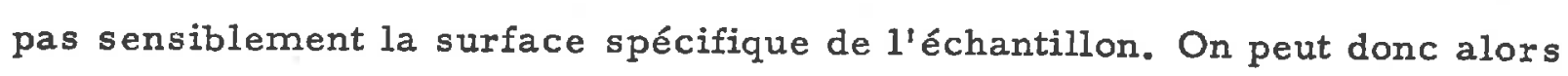
s'interroger sur les raisons qui font que; dans une espèce minérale donnée, suivant ses propriétés cristallochimiques, les mailles élémentaires s'as semblent suivant une, deux ou trois dimensions de l'espace.

Les différentes hypothèses présentées à ce sujet ont été rassemblées et discutées par PEDRO dans son ouvrage sur la classification des minéraux argileux. Oiı pourrait considérer à la base la nature des liaisons. Liaisons H pour les kaolinites, liaisons ioniques dans le cas des micas et enfin liaisons ioniques par cations hydratés pour les vermiculites et les smectites. Suivant la répartition des riéficits de charge entrainant la liaison ionique, les feuillets 
s'ássocieraient alors suivant un ordre plus ou moins rigoureux et une juxtaposition plus ou moins étroite des feuillets.

C'est ainsi que l'absence de déficit de charge sur les surfaces basales des minéraux de types kaolinite, devrait faciliter la superposition des feuillets qui s'associeraient d'autre part par une liaison hydrogène. Cette possibilité aboutirait ainsi à former des minéraux "épais", donc à faible surface spécifique. I,'existence d'un déficit de charge plus ou moins ordonné assurerait égajement une association face contre face des feuillets des micas et de l'illite conduisant également à des assemblages d'une certaine épaisseur. Au contraire dans le cas des smectites, un certain désordre dans la répartition des charges, la position de çentre d'attraction au niveau de la couche octaédrique facilitant la dissociation des cations adsorbés, s'opposerait au contraire à la s uperposiiion régulière des feuillets et conduirait ainsi à des très grandes surfaces spécifiques.

On pourrait également associer à ces considérations, les propriétés du milieu où le minéral prend naissance. Suivant sa composition ionique, sa concentration, la présence de certains ions, leurs variétés, pourraient également s'opposer à la disposition régulière et par conséquent à la juxtaposition étroite des feuillets constituant le cristallite.

Ce n'est évidemment pas en partant des relations concernant la surface que l'on peut espérer résoudre le problème de la croissance. En Évoquant ici ces diverses possibilités, notre but était seulement de montrer que l'on pourait déjà concevoir, dans l'état actuel de nos connaissances, une rulation cuntre les possibilités de croissance et les propriétés cristallochimiques 
des minéraux des argiles. Les réflexions que nous avons présentées montrent en effet que c'est sur cette base que l'on doit interpréter la relation qui pourrait exister entre la surface spécifique et la nature du minéral.

Manuscrit reçu le 30 Mai 1969 